\title{
AVALIAÇÃO DE IMPACTOS AMBIENTAIS PRÉ-OPERACIONAIS EM PROJETOS DE EDIFICAÇÕES E A MODELAGEM DA INFORMAÇÃO DA CONSTRUÇÃO'
}

\author{
EVALUATION OF ENVIRONMENTAL PRE-OPERATION IMPACTS IN \\ BUILDING PROJECTS AND THE BUILDING INFORMATION MODELING
}

\author{
Eloise de Oliveira \\ Universidade Federal do Paraná (UFPR) \\ eloisedeoliveira@gmail.com \\ Sergio Scheer \\ Universidade Federal do Paraná (UFPR) \\ sergioscheer@gmail.com \\ Sergio Fernando Tavares \\ Universidade Federal do Paraná (UFPR) \\ sergioftavares@gmail.com
}

\begin{abstract}
Resumo
Atualmente a grande demanda por edificações mais sustentáveis se justifica na busca pelo desenvolvimento sustentável e nos impactos ambientais associados às atividades da construção civil. Através dos recursos computacionais BIM (Building Information Modeling) é possível prever o comportamento de uma edificação ainda em fase de projeto e realizar alterações para o alcance da sustentabilidade. Este trabalho busca explorar as formas de integração entre o processo de modelagem BIM e as análises energéticas de edificações. A avaliação de impactos ambientais em edificações na fase pré-operacional destaca-se como uma forma prática de mitigar estes impactos nas edificações. Para essa discussão teórica, foi utilizado o método de revisão bibliográfica. Entre as publicações mais recentes sobre a aplicabilidade do BIM para medir a sustentabilidade de edificações e a norma brasileira para modelagem, foram analisados os trabalhos a respeito da metodologia de Análise do Ciclo de Vida em edificações. Com base na bibliografia revisada observa-se que os esforços para avaliar aspectos de sustentabilidade em BIM estão direcionados a uma abordagem com pontos de incerteza e limitações. Há uma necessidade de aperfeiçoamento metodológico e operacional dos procedimentos existentes, bem como uma nova perspectiva de Análise Energética focada nos impactos ambientais inerentes à etapa pré-operacional das edificações.
\end{abstract}

Palavras-chave: Building Information Modeling. Sustentabilidade. Análise Energética. Energia Embutida.

\begin{abstract}
Currently, the high demand for sustainable buildings justifies the efforts to achieve the sustainable development and the environmental impacts associated with the activities of construction sector. The building information modeling (BIM) can predict the behavior of a building in design stage and suggest alteration to achieve the
\end{abstract}

1 OLIVEIRA, E.; SCHEER, S.; TAVARES, S. Avaliação de impactos ambientais pré-operacionais em projetos de edificações e a modelagem da informação da construção. In: ENCONTRO BRASILEIRO DE TECNOLOGIA DE INFORMAÇÃO E COMUNICAÇÃO NA CONSTRUÇÃO, 7., 2015, Recife. Anais... Porto Alegre: ANTAC, 2015. 
sustainability. This study aims to explore the ways of integrations between the process of BIM and the building energy analyses. The building assessment of environmental impacts realized in the preoperational phase of building stands out as practical way to mitigate environmental impacts associate with buildings. For this theoretical discussion, we used a literature review method. Among the lasted publications on the applicability of BIM to measure the sustainability of buildings and the Brazilian standard to modeling studies, ware analyzed studies about the life cycle assessment methodology in buildings. Based on literature reviewed is observed that efforts to evaluate aspects of sustainability with BIM tools are directed to an approach with points of uncertainty and limitations. There is a need for methodological and operational improvement of exist procedures, as well as new perspective of energy analyses, focusing on the impacts arising from preoperational stage of buildings.

Keywords: Building Information Modeling. Sustainability. Energy Analysis. Embodied Energy.

\section{INTRODUÇÃO}

Nos últimos anos o termo Building Information Modeling - BIM vem se tornando cada vez mais comum na indústria da construção, ainda que, muitas vezes, seja associado a uma topologia de softwares e não à um processo de trabalho. No entanto, a nomenclatura e o conceito BIM não são novos, em 1975, Chuck Eastman publicou um trabalho que incluiu as primeiras definições do processo (EASTMAN, 1975). Entre as principais características desse método destaca-se a abordagem do ciclo de vida das edificações, permitindo análises preliminares do comportamento da edificação em todas as etapas do ciclo. Esta abordagem se aproxima dos estudos de impactos ambientais associados às atividades da construção civil. Através da análise do ciclo de vida das edificações - é possível identificar e mitigar impactos diretos e indiretos inerentes ao processo de construção das edificações.

Em países desenvolvidos e em desenvolvimento as discussões sobre os impactos ambientais associados à construção civil estão tomando força com o objetivo de buscar novas soluções para minimizar os motivadores das mudanças climáticas. Dentre os principais causadores do aumento do nível dos gases de efeito estufa estão as atividades econômicas que demandam grande quantidade de energia não renovável. Apenas o setor da construção civil representa $24 \%$ da extração global de matéria prima (BRIBIÁN; CAPILLA; USÓN, 2011). Esse dado se fundamenta na metodologia de análise do ciclo de vida em edificações, a qual considera os processos de fabricação dos materiais de construção, transporte até o canteiro, produção da edificação, uso e manutenção, demolição e disposição final dos materiais utilizados. Esta abordagem do processo fabricação das edificações estabelece uma clara relação entre o método de trabalho proposto pelo BIM 6D, o qual pode-se acrescentar noções de sustentabilidade e alcançar uma definição mais ampla desta dimensão.

Os dimensões BIM partem da modelagem 3D e atualmente incluem variáveis de tempo (4D), custo (5D) e operação da edificação (6D). Segundo Smith (2014), a dimensão 4D conectas as informações do modelo 3D com dados de cronograma de execução de obra, facilitando a simulação das atividades de construção. O BIM 5D agrega todas as informações do modelo 3D e 4D com dados de custo, fornecendo o quantitativo de materiais e encargos de obra. A sexta dimensão BIM representa o modelo construído e constitui a chamada facilities management, ou seja, o gerenciamento do ciclo de vida da edificação. Considerando a perspectiva agregada pelo BIM 6D pode-se acrescentar definições de sustentabilidade e alcançar uma aplicabilidade mais ampla desta dimensão, incluindo novos parâmetros e expandindo os limites de informação do modelo.

Através de referenciais teóricos, esta pesquisa tem como objetivo explorar as formas de colaboração da modelagem da informação da construção para a mitigação dos impactos ambientais associados às atividades da construção civil. 


\section{CICLO DE VIDA DAS EDIFICAÇÕES E OS IMPACTOS AMBIENTAIS ASSOCIADOS À CONSTRUÇÃO CIVIL}

Os impactos ambientais associados à construção civil podem ser avaliados através de um método de Análise do Ciclo de Vida - ACV adaptado às edificações, a partir deste estudo encontra-se os valores de Energia Embutida - EE e emissão de $\mathrm{CO}_{2}$ equivalente. Os estudos de ACV fazem uma abordagem de toda a vida de um produto e estão regulamentados pela norma internacional ISO 14040:2006 que funciona como um protocolo e define as quatro fases chaves da aplicação do método; primeiramente deve-se estabelecer o objetivo e escopo, depois realizar uma análise do inventário levantado, escolher uma categoria de impacto ambiental e interpretar dos resultados (MONCASTER, A.M., SYMONS, K.E., 2013). Para auxiliar os profissionais na condução dos estudos de ACV existem softwares com protocolos próprios e base de dados disponíveis para utilização pública.

A metodologia de análise do ciclo de vida de edificações aborda o ciclo de vida completo, considerando desde a extração e fabricação dos materiais de construção, transporte até o canteiro, produção da edificação, uso e manutenção durante a vida útil e demolição e disposição dos materiais empregados. Em todas as etapas existe um requisito de energia e emissão de $\mathrm{CO}_{2}$ para a sua realização, na Figura 1 estão representadas as etapas consideradas neste ciclo.

Figura 1 - O ciclo de vida das edificações

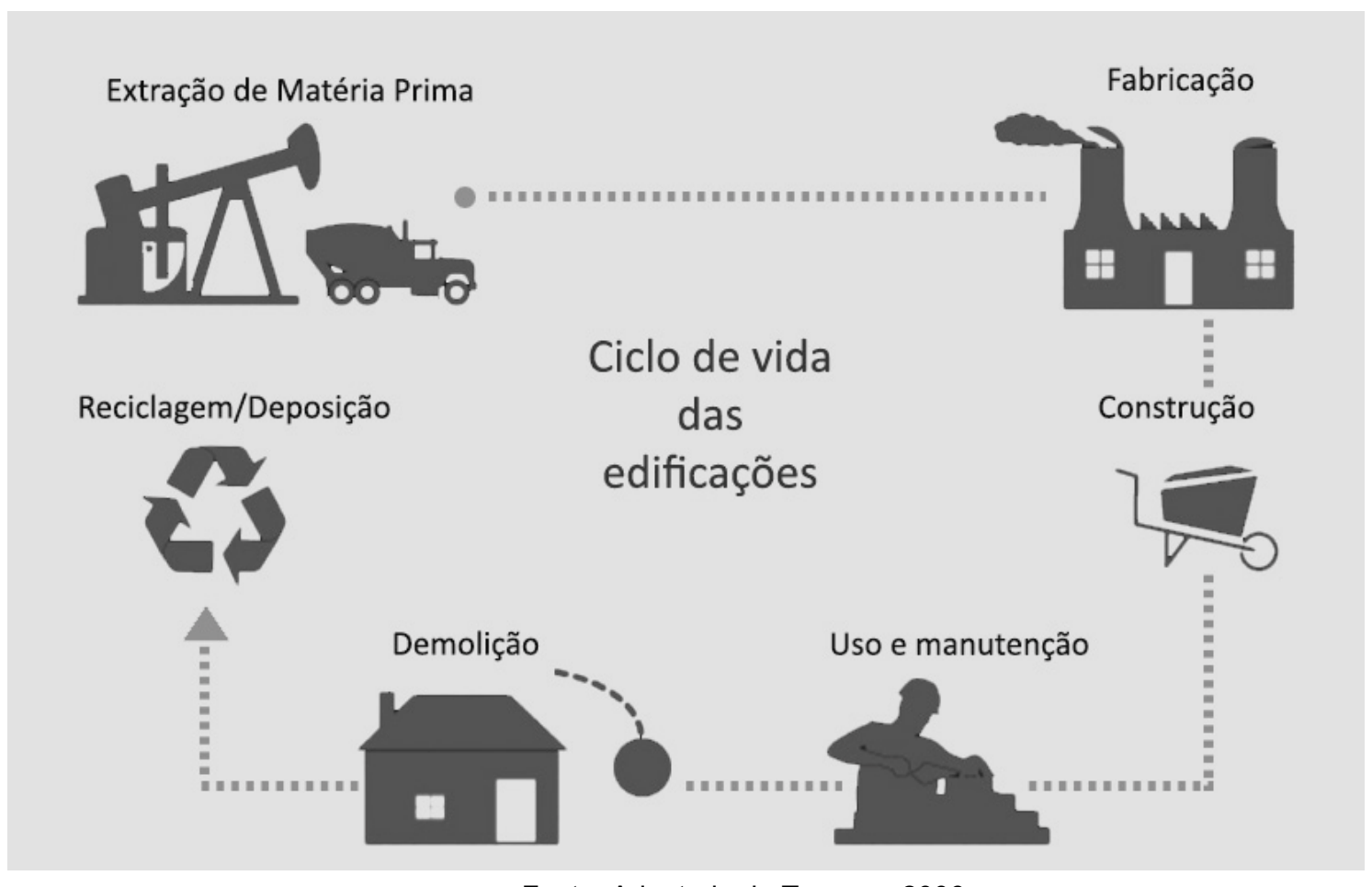

Fonte: Adaptado de Tavares, 2006

Na metodologia de ACV, o ciclo de vida das edificações é dividido em três etapas: préoperacional, operacional e pós-operacional. Considera-se como pré-operacional toda a energia requisitada para a construção da edificação, a chamada EE - Energia Embutida. A etapa operacional contabiliza todos os gastos energéticos para o funcionamento da edificação e valores de EE de manutenção e, a fase pós-operacional considera a energia para demolição e disposição dos resíduos. 


\section{ANÁLISE ENERGÉTICA PRELIMINAR EM EDIFICAÇÕES}

As análises energéticas preliminares podem estar associadas a duas abordagens distintas, cada qual em uma etapa do ciclo de vida das edificações. De acordo com Tavares (2006), a análise energética preliminar está associada à energia embutida na fase pré-operacional da edificação, ou seja, no total de requisitos energéticos utilizados na produção dos materiais e transporte dos materiais até o canteiro. No entanto, algum autores entendem como análise preliminar o processo de simulação e avaliação dos consumo energético ao longo da vida útil da edificação. Ambas as abordagem configuram um estudo de uma das partes do ciclo de vida da edificações e, é importante destacar que em todos os processos existe um impacto ambiental associado. Segundo Tavares (2006) a energia requisitada na fase préoperacional representa $35 \%$ do total consumido por uma residência típica brasileira em 50 anos, enquanto a fase operacional representa $59 \%$.

Considerando o baixo consumo energético para o aquecimento e resfriamento das edificações no Brasil e a matriz energética brasileira que possui $85 \%$ de energia originada de fontes renováveis (BRASIL, 2013), pode-se considerar que o consumo energético na fase operacional da edificação possui um impacto razoável, se considerarmos os impactos causados pela fabricação de materiais empregados na construção das edificações. Em 2013 foram produzidos mais de 70 milhões de toneladas de cimento no país (CBIC, 2013), sendo este material, um importante contribuinte nas emissões anuais de $\mathrm{CO}_{2}$. De acordo com as estimativas de emissão de gases do efeito estufa no Brasil, o cimento detém $26,9 \%$ do total de $\mathrm{CO}_{2}$ emitido pelas atividades industriais (BRASIL, 2006). O impacto ambiental associado aos materiais está diretamente relacionado à origem da energia empregada na sua produção. Através do Quadro 1 é apresentada uma síntese das fontes utilizadas para a fabricação dos principais materiais de construção.

Quadro 1 - Fontes de origem da energia no setor industrial para fabricação de materiais de construção civil

\begin{tabular}{|l|l|l|}
\hline Setor Industrial & Fontes Fósseis* & $\begin{array}{l}\text { Fontes } \\
\text { Renováveis** }\end{array}$ \\
\hline Cimento & 84.6 & 15.4 \\
\hline Ferro-gusa e aço & 67.36 & 32.74 \\
\hline Ferroligas & 26.6 & 73.5 \\
\hline Mineração e pelotização & 76.88 & 23.12 \\
\hline Não ferrosos e outros metálicos & 54.96 & 45.04 \\
\hline Papel e celulose & 71.02 & 28.98 \\
\hline Cerâmica & 43.14 & 56.76 \\
\hline $\begin{array}{l}\text { Outras indústrias } \\
\text { * Fontes de origem fóssil consideradas: carvão mineral, óleo combustível, eletricidade } \\
\text { oriunda de usinas térmicas e nucleares (20\%), coque de petróleo, gás natural e outras. } \\
\text { ** Fontes de origem renovável consideradas: eletricidade oriunda de usinas hidroelétricas } \\
\text { (80\%), carvão vegetal. }\end{array}$ & 48.64 \\
\hline
\end{tabular}


É importante considerar outros fatores que determinam o impacto ambiental associado a um material e como ele deverá ser avaliado, havendo diferentes categorias de impactos relacionadas à produção e ao uso. A emissão de gases geradores do efeito estufa resultante do processo de produção é um dos protocolos mais utilizados no estudo do ciclo de vida de produtos. Outra variante que determina o impacto dos materiais é a maneira na qual eles serão incorporados ao projeto (ROAF, 2014), sendo assim as análises energéticas preliminares que avaliam a energia embutida inicial na edificação poderão ser utilizadas como ferramenta de tomada de decisões durante a fase preliminar de desenvolvimento do projeto, assim como a análise da energia operacional poderá ser utilizada para a simulação do comportamento na fase operacional da edificação. Para um melhor entendimento, os tópicos seguintes descrevem a abordagem das análises nas diferentes etapa das do ciclo de vida da edificação.

\subsection{Energia Embutida Inicial}

Qualquer atividade de transformação ou transporte de matéria implica em uso de uma forma de energia. E a determinação da energia requerida para a produção de um bem ou serviço, requer uma forma sistematizada de avaliação que pode ser chamada de análise energética (TAVARES, 2006). Estas atividades de transformação de matéria prima envolvem extração, manufatura e transporte, e possuem energia incorporada em cada um desses processos. Esta energia pode ser avaliada pelo estudo de análise energética e assim obter a energia embutida no bem ou serviço analisado. Segundo Tavares (2006), o conceito de EE, seria o total dos requisitos energéticos para a produção de determinado material. Utilizar este termo é também um modo de simplificar o método de análise energética para a sua aplicação em edificações.

Para Bribián, Capilla e Usón (2011), os edifícios são considerados produtos complexos, pois têm uma vida relativamente longa, sofrem constantes alterações, muitas vezes possuem múltiplas funções e contêm muitos componentes distintos. Logo, o objetivo de avaliar a EE é encontrar os requisitos de energia empregados na produção destes componentes, nas edificações é a soma dos materiais utilizados na sua produção pela energia incorporada na produção de cada material. Esta abordagem de análise energética preliminar corresponde à primeira fase do ciclo de vida energético da edificação, a pré-operacional. $\mathrm{Na}$ etapa operacional da edificação também são contabilizados os dados de energia embutida resultantes da manutenção, substituição e reposição de materiais ao longo da vida útil do edifício.

A produção de materiais para a construção civil além ter uma alta demanda energética, possui elevados índices de emissão de $\mathrm{CO}_{2}$ nos processo de fabricação. Por este motivo há grandes esforços para que as Análises Energéticas sejam utilizadas por arquitetos e engenheiros em uma fase preliminar do projeto das edificações, quando há a possibilidade de considerar diferentes materiais, com variáveis de energia embutida e emissão de $\mathrm{CO}_{2}$. Nas Figuras 2 e 3 são demostradas as contribuições relativas dos principais materiais de construção para EE e emissões de $\mathrm{CO}_{2}$ associada à $1 \mathrm{~m}^{2}$ de uma unidade residencial. 
Figura 2 - Demanda de energia primaria para manufatura dos materiais necessários para construção de $1 \mathrm{~m}^{2}$

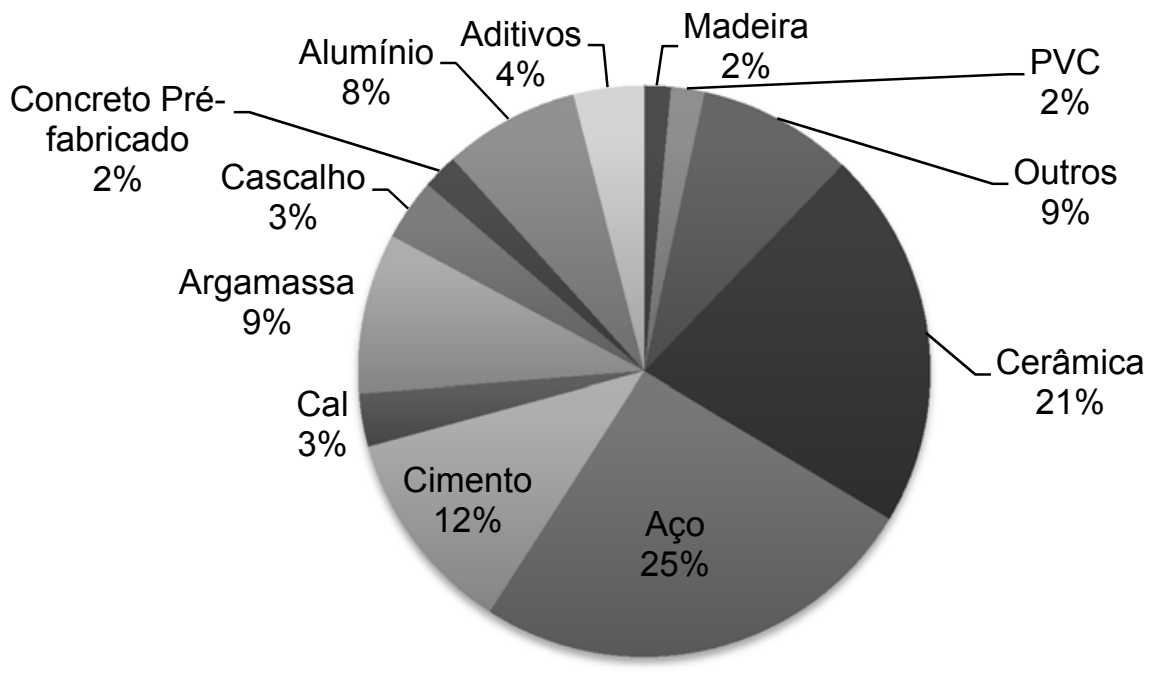

Fonte: Adaptado de BRIBIÁN; CAPILLA; USÓN (2011)

Figura 3 - Contribuição de $\mathrm{CO}_{2}$ associada ao processo de manufatura dos materiais necessários para construção de $1 \mathrm{~m}^{2}$

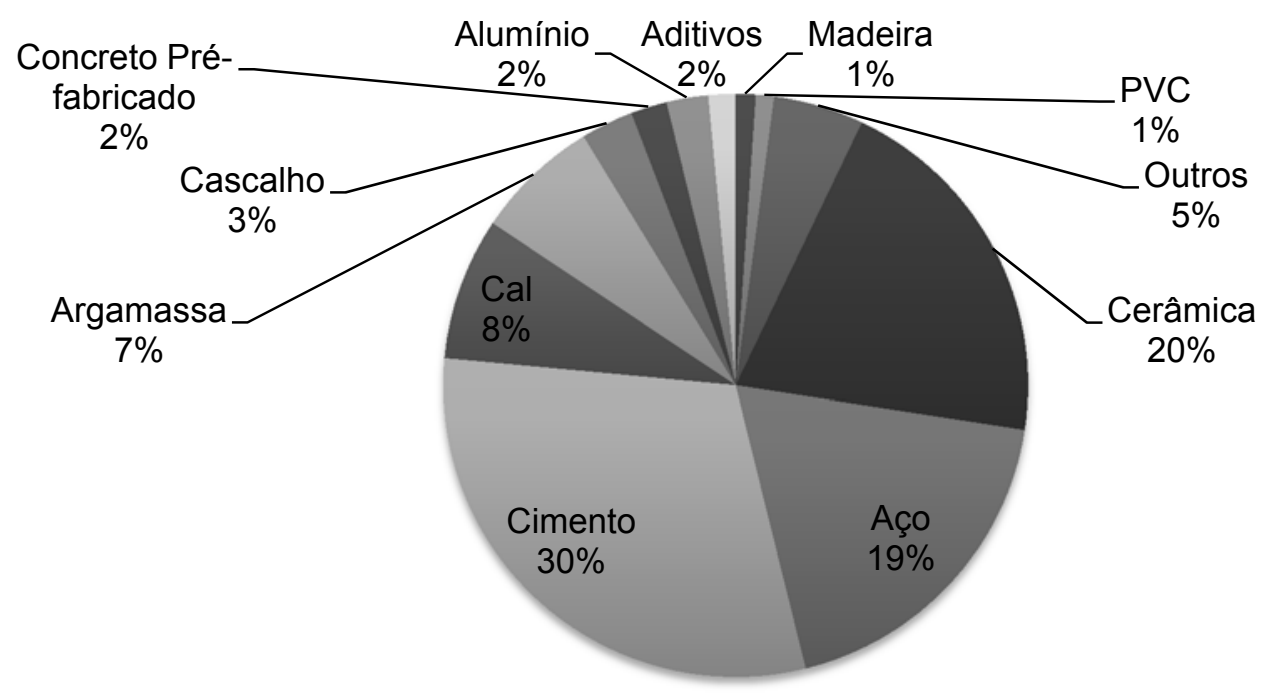

Fonte: Adaptado de BRIBIÁN; CAPILLA; USÓN (2011)

Acredita-se que para melhorar o desempenho energético das edificações brasileiras, é preciso incentivar novas metodologias de desenvolvimento de projeto com o objetivo de reduzir a Energia Embutida inicial e de manutenção e a emissão de $\mathrm{CO}_{2}$ ao longo do ciclo de vida da edificação. $O$ design aliado à processos integrados de projeto pode se mostrar uma forma eficaz de reduzir tempo, custo e, também, os impactos ambientais associados às edificações. 


\section{Q1}

\subsection{Energia Operacional}

Tavares (2006) descreve a energia operacional como aquela consumida durante a vida útil da edificação para suprir necessidades de climatização, iluminação, entretenimento, cocção ou atividades profissionais. A energia operacional varia conforme o uso da edificação, as condições climáticas e o nível de conforto exigido. Existem três setores que estão ligados ao consumo energético na fase operacional das edificações: comercial, residencial e público. De todos os setores, o residencial possui o maior consumo de recursos energético no Brasil, segundo o BEN - Balanço Energético Nacional (BEN 2013). Na Figura 4 é ilustrado o consumo residencial brasileiro por fonte.

Figura 4: Consumo no setor residencial

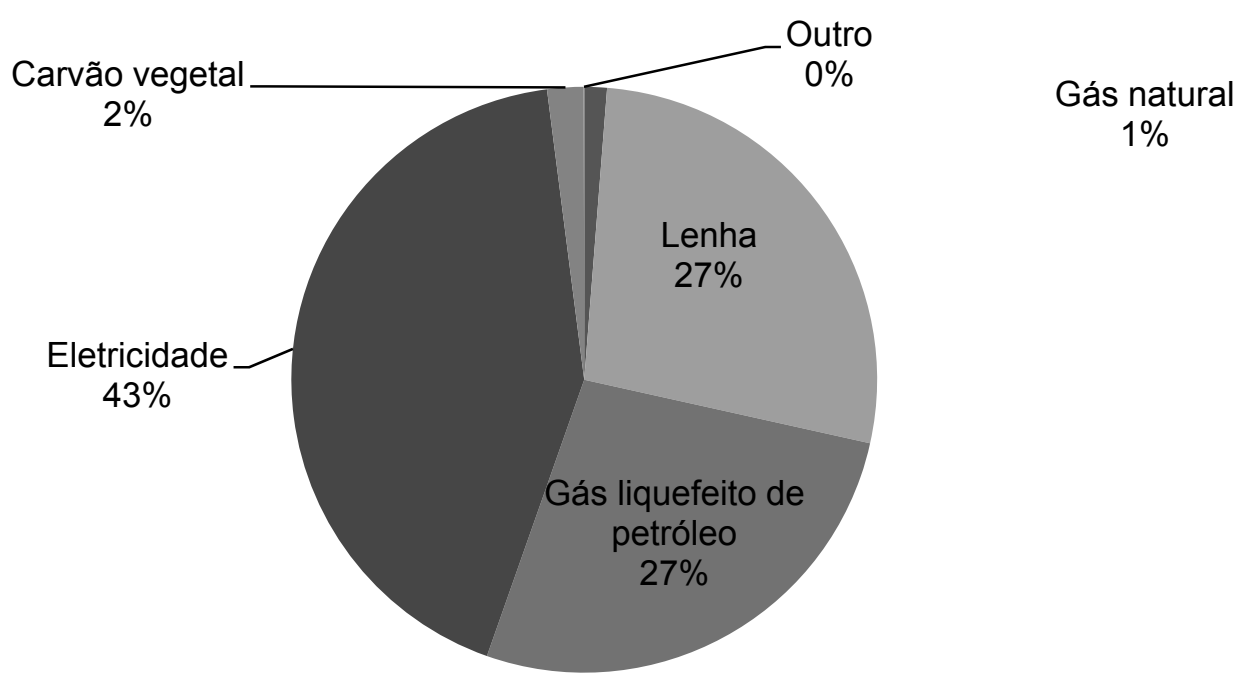

Fonte: BEN, 2013

É importante considerar o recente aumento no número de novas residências no Brasil, decorrente de incentivos econômicos para suprir o déficit de moradias no país e melhor equipar as residências. Esse processo resulta no aumento da demanda energética no setor residencial brasileiro. Com base nesta realidade também é importante afirmar a falta de aprofundamento nas questões ambientais na maioria dos projetos replicados em grande escala. Além da falta de soluções para questões de ventilação passiva e aproveitamento de luz natural, como produtos, muitos apresentam um baixa qualidade em função do processo de execução, resultando em unidades habitacionais com alto consumo energético e intensa necessidade de manutenção. Esses quesitos elevam o consumo de recursos naturais pelas edificações e contribuem para os impactos ambientais associados ao setor da construção civil.

\section{BUILDING INFORMATION MODELING}

A metodologia de trabalho BIM tem como objetivo integrar profissionais na elaboração de um modelo virtual inteligente. Um modelo BIM possui modelagem paramétrica, orientação ao objeto, usabilidade em todo o ciclo de vida do projeto, colaboração, interoperabilidade e uma biblioteca de componente. Quando completo, o modelo gerado computacionalmente contém a geometria exata e os dados necessários para dar suporte à construção, à fabricação e ao fornecimento dos insumos necessários para a realização da construção 
(EASTMAN, 2014). A utilização da tecnologia BIM no processo de projeto ocorre através da construção do modelo virtual a partir de objetos que simulam o comportamento dos elementos construtivos, o modelo é uma composição de objetos inteligentes que carregam suas próprias informações e podem ser entendidos como bases de dados onde são armazenados tanto os dados geométricos, como os textuais de cada elemento construtivo utilizado no projeto (SCHEER et al., 2007).

Uma das vantagem da utilização da modelagem é que a partir da elaboração de um único modelo são extraídos automaticamente desenhos técnicos como plantas, cortes, elevações, perspectivas, detalhamentos e dados quantitativos referente a tempo e custo da obra. $\mathrm{O}$ processo torna-se mais ágil e preciso, eliminando o retrabalho e minimizando erros de projeto por compatibilização, já que uma única alteração no modelo gera uma atualização nos demais dados obtidos a partir dele. Desta forma, o processo de desenvolvimento do projeto arquitetônico torna-se mais focado nas soluções de projeto que serão adotas pelo arquiteto, também permite que o profissional verifique previamente se o projeto está adequado à realidade do meio em que será construído. Esses fatores melhoram a qualidade do projeto, assim como conferem maior precisão e segurança na execução do produto final, através da integração e troca de informações necessárias para a elaboração, execução e manutenção do empreendimento.

O trabalho realizado por Succar (2009) apresenta as possibilidades de expansão da tecnologia BIM através da adição de lentes e filtros que representam a terceira dimensão da metodologia. As lentes são camadas que permitem uma visualização profunda do conhecimento, já os filtros removem os detalhes desnecessários dessa análise. Segundo Succar, as lentes são ferramentas de investigação que possibilitam que pesquisadores foquem em um determinado aspecto da indústria da construção e gerem conhecimento e diferentes pontos de vista. Nesse trabalho, busca-se aplicar uma lente com aspectos de sustentabilidade.

\section{UTILIZAÇÃO DA MODELAGEM DA INFORMAÇÃO DA CONSTRUÇÃO PARA A AVALIAÇÃO DE IMPACTOS AMBIENTAIS ASSOCIADOS ÀS EDIFICAÇÕES}

Com o recente aumento na demanda por edificações mais sustentáveis, profissionais ligados ao setor da construção civil têm buscado ferramentas que auxiliam a tomada de decisões projetuais com o objetivo de minimizar os impactos ambientais associados às edificações. Acredita-se que as decisões mais eficazes, em termos de redução de impactos ambientais, devem ser tomadas na fase preliminar de desenvolvimentos do projeto arquitetônico. Sendo assim, o BIM tem sido visto como um importante colaborador para a adoção de medidas de sustentabilidade incorporadas desde o início do projeto da edificação. As informações multidisciplinares contidas em um único modelo permitem que uma grande variedade de soluções de projeto sejam avaliadas rapidamente, auxiliando os profissionais no processo de tomada de decisão. Neste trabalho, foram levantados estudos que verificam diferentes formas de utilização do BIM para avaliações de sustentabilidade em edificações.

\subsection{Limitações das simulações de desempenho energético em edificações}

O trabalho realizado por Mile et al. (2010) investiga e documenta as limitações das simulações energéticas. Os autores atribuem as diferenças entre o desempenho energético medido na edificação e o simulado à aproximações, suposições e simplificações (ASS) no processo de simulação. A definição de aproximação é relacionada à valores matemáticos, a suposição é vinculada à falta de questionamento e a simplificação à adoção de processos demasiadamente simplificados. Com base na literatura existente, os autores desenvolveram categorias para listar os problemas de ASS mais recorrentes nas simulações energéticas. 
As nove categorias estão fundamentadas em problemas de entrada de dados, construção do modelo e dados de uso da edificação.

O mecanismo de simulação escolhido para o estudo de quatro casos foi o EnergyPlus, um software que possui interoperabilidade com ferramentas BIM através da habilidade de criar modelos baseados na linguagem Industry Foundation Classes (IFC). Os principais pontos de incerteza encontrados nas simulações estão relacionados à representação geométrica inadequada, incapacidade de gerar objetos, sistemas e configurações únicos, limitações de comparação de dados de medição, limitações de interoperabilidade, dificuldade com a interface gráfica e aumento no nível de detalhes do modelo.

Os autores apontam para a necessidade de melhorias relacionadas à interoperabilidade, com o objetivo facilitar o intercâmbio de dados e aumentar a prática da simulação baseada no modelo arquitetônico da edificação. A eficiência da troca de dados permitiria elevar o nível de detalhamento do projeto, reduzir os pontos de incertezas e atualizar rapidamente o nível de eficiência do modelo.

\subsection{Procedimentos de simulação da NBR 15575}

Sorgato et al. (2014) realizam uma avaliação do procedimento indicado pela NBR 15575-1 para simulação de desempenho térmico de edificações residenciais. O estudo compara os resultados de desempenho térmico de uma residência obtidos através do procedimento de simulação indicada pela norma com uma metodologia alternativa. Ao utilizar o software EnergyPlus os autores analisam a influência das condições do piso da edificação em contato com o solo e, como resultado, os procedimentos indicam que tais condições exercem forte influência no desempenho térmico da edificação. Ao comparar os procedimentos, os autores observam que há uma grande diferença entre os resultados obtidos. Atribui-se esse fato a parâmetros, de cargas térmicas internas, condições de contato com o solo e ventilação natural, não considerados pela norma.

Silva et al. (2014) também investigam as incertezas do método de simulação da NBR 15575-1, utilizando o software EnergyPlus. Como resultado, os autores constatam que as variáveis relacionadas à data do dia típico de verão e inverno, à velocidade e direção do vento, o algoritmo de cálculo de irradiação solar e tipo de céu, exercem grande influência nos resultados e nos níveis de classificação do desempenho térmico da edificação, causando falta de precisão no resultado final. Tais variáveis são desconsideradas pela norma e, por isso, podem ser facilmente alteradas para obter uma melhor classificação do nível de desempenho da edificação.

Observa-se que além dos problemas metodológicos e pontos de incerteza levantados pelos trabalhos de Sorgato et al. (2014) e Silva et al. (2014), a norma não considera a etapa préoperacional do ciclo de vida da edificação. Ao desconsiderar a EE inicial a metodologia ignora impactos significativos e conduz uma avaliação a partir de um modelo ou projeto em fase final de desenvolvimento, permitindo pouca possibilidade de alteração projetual antes da execução da obra da edificação.

\subsection{Avaliação de impactos ambientais através de um sistema CAD-BIM}

Basbagill et al. (2013) sugerem a utilização da ACV na etapa preliminar de desenvolvimento de projeto, com o objetivo de fornecer um feedback sobre os impactos ambientais associados às escolhas aplicadas ao modelo BIM. O método informa ao profissional os componentes da edificação que possuem maior impacto ambiental associado e, através da sugestão de materiais e dimensionamentos para a execução dos elementos construtivos, apresenta um plano de redução de impactos. O escopo da metodologia considera as etapas pré-operacional e operacional do ciclo de vida da edificação através da integração de um 
software BIM com a metodologia de $\mathrm{ACV}$ e a análise energética por software. A análise de sensibilidade é realizada com base nos resultados das simulações e tem como objetivo apontar quais componentes de construção contribuem de forma consistente para o impacto ambiental da edificação.

Para Heiselberg e Brohuns (2008) a análise de sensibilidade determina a variação de contribuição do detalhe individual para o desempenho total da solução de projeto. Segundo os autores, as análises de sensibilidade podem ser agrupadas em três classes: métodos de triagem, métodos de sensibilidade local e métodos de sensibilidade global. A primeira classe é direcionada à situações complexas as quais possuem alto custo computacional ou grande número de parâmetros de projeto. Os métodos de triagem utilizam valores padrão como controle e são uma forma econômica de identificar e classificar qualitativamente os parâmetros de projeto que detém a maior parte da variabilidade do desempenho energético. Os métodos de sensibilidade local possuem uma abordagem seccionada, considerando a análise de um parâmetro por vez, enquanto os demais parâmetros de projeto são mantidos constantes. Neste tipo de análise não é considerada a correlação entre os parâmetros de concepção de projeto. Os métodos de sensibilidade global associam a variabilidade de todos os parâmetros de projeto e consideram a influência entre parâmetros para avaliar o desempenho geral do edifício. Este método é dependente da inter-relação entre todos os parâmetros, uma vez que o objetivo da análise de sensibilidade é avaliar o edifício como um todo e não apenas um parâmetro em individual.

No cenário brasileiro, Ayres (2009) propõe a utilização de uma ferramenta denominada EEQuant, a qual permite avaliar a quantidade de EE e Emissão de $\mathrm{CO}_{2}$ nos materiais da edificação, ainda em fase preliminar de projeto. O corpo principal da ferramenta EEQuant é justamente uma base de dados auxiliar, na qual foram inseridos (como componentes) os dados relativos ao consumo de energia e emissão de $\mathrm{CO}_{2}$ na produção e no transporte dos materiais de construção, essa inserção ocorreu em uma janela de edição do ArchiCAD (Ayres, 2009). A ferramenta EEQuant permite que o projetista avalie, de forma rápida, diferentes possibilidades de materiais e os impactos ambientais associados à cada solução projetual adotada. A principal desvantagem, apontada pelo autor, é que a ferramenta limitase à estrutura disponibilizada pelo software para inserção de dados. No entanto, o trabalho de Ayres (2009) comprova a viabilidade de utilizar uma ferramenta BIM para avaliações de sustentabilidade em edificações, no tocante da $\mathrm{EE}$ e $\mathrm{CO}_{2}$ Embutido na fase pré-operacional da edificação.

\section{CONCLUSÕES}

Observa-se que existe uma grande quantidade de consumo energético e emissão de $\mathrm{CO}_{2}$ durante todo o ciclo de vida das edificações e que o momento mais adequado para tomar decisões com o objetivo de minimizar os impactos ambientais é na etapa preliminar de concepção projetual. No entanto, este trabalho demonstrou que as pesquisas mais recentes realizam análises energéticas no final do desenvolvimento do projeto, esses procedimentos buscam simular o comportamento energético da edificação durante a fase de uso e operação, descartando a energia requisitada e os altos índices de emissão de $\mathrm{CO}_{2}$ na produção dos materiais, transporte até o canteiro e na construção da edificação. Essa prática resulta em um processo de retrabalho e minimiza as oportunidades de utilizar materiais mais eficientes e com menor impacto ambiental associado à sua fabricação, reduzindo consideravelmente a $\mathrm{EE}$ e o $\mathrm{CO}_{2}$ incorporado na edificação.

No entanto pode-se afirmar que através de recursos computacionais de simulação somado ao processo BIM, é possível prever o comportamento de uma edificação ainda em fase de projeto e realizar alterações para o alcance de edificações mais sustentáveis. Contudo, as simulações de análise energética ainda apresentam dificuldades de entrada e interpretação 
de dados e interoperabilidade. Sendo assim, este trabalho buscou explorar as formas de integração entre o processo de modelagem BIM e as análises energéticas de edificações. A avaliação de impactos ambientais em edificações na fase pré-operacional destacou-se como uma forma prática de mitigar os impactos ambientais associados às edificações.

A partir da fundamentação teórica estabeleceu-se uma conexão entre as metodologias BIM e ACV. Foram levantados estudos que relatam diferentes limitações de análises energéticas por simulação e exemplos que consideram a Análise Energética Preliminar através da avaliação de EE e $\mathrm{CO}_{2}$ incorporado como base de dados de uma ferramenta BIM. Ainda que as ferramentas BIM permitam realizar uma avaliação de sustentabilidade, entre outras informações multidisciplinares, notou-se a sua pouca utilização devido às divergências entre resultados de simulação e dados reais. Com base na bibliografia revisada observou-se que os esforços para avaliar aspectos de sustentabilidade em BIM estão direcionados à uma abordagem com pontos de incerteza e limitações. É possível afirmar que há uma necessidade de aperfeiçoamento metodológico e operacional dos procedimentos existentes, bem como uma nova perspectiva de Análise Energética focada nos impactos ambientais inerentes às etapas pré-operacionais das edificações. Este trabalho é uma contribuição inicial para aprimorar as formas de verificar a sustentabilidade em edificações através do BIM. Conclui-se que é de grande importância a inclusão de aspectos de sustentabilidade à metodologia de modelagem da informação da construção, com o objetivo de viabilizar a utilização desse tipo de análise por profissionais da construção, além elevar a qualidade dos projetos em função das demais características da tecnologia BIM.

\section{REFERÊNCIAS}

AYRES, C. F. Acesso ao modelo integrado do edifício. Dissertação (Mestrado em Engenharia de Construção Civil) - Setor de Exatas, Universidade Federal do Paraná, Curitiba, 2009.

BASBAGILL, J.; FLAGER, F.; FISCHER, M. Application of life-cycle assessment to early stage building design for reduced embodied environmental impacts. Building and Environment, v. 60, p. 81-92. Elsevier Science Ltd. 2013.

Brasil, Ministério de Minas e Energia. 2006. Estimativas anuais de emissões de gases do efeito estufa no Brasil. Brasília.

Brasil, Câmara Brasileira da Industria da Construção. 2013. Produção Mensal de Cimento - por UF, Grande Regiões e Totais no Brasil. Brasília.

Brasil, Ministério de Minas e Energia. 2013. Balanço Energético Nacional. Brasília

BRIBIÁN, I. Z.; CAPILLA, A. V.; USÓN A. A Life cycle assessment of building materials: Comparative analysis of energy and environmental impacts and evaluation of the ecoefficiency improvement potential. Building and Environment, v. 46, p. 1133-1140. Elsevier Science Ltd. 2011.

EASTMAN, C. Spatial Synthesis in Computer-Aided Building Design. Nova York: Elsevier Science, 1975.

EASTMAN, C.; TEICHOLZ, P.; SACKS, R.; LISTON, K. Manual de BIM - Um guia de modelagem da informação da construção para arquitetos, engenheiros, gerentes, construtores e incorporadores. Porto Alegre: Bookman, 2014. 
GRAF, H. F.; TAVARES, S. F. Energia Incorporada dos Materiais de uma Edificação Padrão Brasileira Residencial. Curitiba: Universidade Tecnológica do Paraná, 2010.

HEISELBERG, P. K.; BROHUS, H. Sensitivity analysis applied in design of low energy office building. In: AIR INFILTRATION AND VENTILATION CENTER CONFERENCE, 29., 2008, Kyoto. Anais... Athens: AIVC, 2008. p. 33-40.

MARCOS, M. H. C.; TAVARES, S. F. Análise de impactos ambientais, na fase préoperacional da edificação, em habitações de interesse social, utilizando ferramenta CAD BIM. In: ENCONTRO NACIONAL E ENCONCTRO LATINOAMERICANO DE EDIFICAÇÕES E COMUNIDADES SUSTENTÁVEIS, 7.,5., 2013, Curitiba. Anais... Porto Alegre: ANTAC, 2013.

MILE, T.; FISCHER, M.; HAYMAKER, J.; BAZJANAC, V. Formalizing approximations, assumptions and simplifications to document limitation in building energy performance simulation. CIFE Working Paper, v. 126. Stanford University, 2010.

MONCASTER, A. M., SYMONS, K. E. A method and tool for "cradle to grave" embodied carbon energy impacts of UK buildings in compliance with the new TC350. Energy and Building, v. 66, p. 514-523. Elsevier Science Ltd. 2013.

ROAF, S. Ecohouse - A casa ambientalmente sustentável. Porto Alegre: Bookman, 2014.

SCHEER, S., ITO, A., AYRES, C., AZUMA, A., BEBER, M. Impactos do uso do sistema CAD geométrico e do uso do sistema CAD-BIM no processo de projeto em escritórios de arquitetura. In: WORKSHOP BRASILEIRO DE GESTÃO DO PROCESSO DE PROJETOS NA CONSTRUÇÃO DE EDIFÍCIOS, 7., 2007, Curitiba. Anais... Curitiba: UFPR, 2007.

SILVA, A. S.; SORGATO, M. J.; MAZZAFERRO, L.; MELO, A. P.; GHISI, E. Incerteza de método de simulação de NBR 15575-1 para avaliação do desempenho térmico de habitações. Ambiente Construído, Porto Alegre, v. 14 , n. 4, p. 103-117, out./dez. 2014.

SMITH, P. BIM \& the 5D project coast manager. In: INTERNATIONAL PROJECT MANAGEMENT ASSOCIATION WORD CONGRESS, 27., 2014, Dubrovnik. Anais... Amsterdam: IPMA, 2014.

SORGATO, M. J.; MELO, A.P.; MARINOSKI, D. L.; LAMBERTS, R. Análise de procedimentos de simulação da NBR 15575 para avaliação de desempenho térmico de edificações residenciais. Ambiente Construído, Porto Alegre, v. 14, n. 4, p. 83-101, out./dez. 2014.

SUCCAR, B. Building information modeling framework: A research and delivery Foundation for industry stakeholders. Automation in Construction, v.15, p.357-375. Elsevier Science Ltd. 2009.

TAVARES, S. F. Metodologia de análise do ciclo de vida energética de edificações residenciais brasileiras. Tese (Doutorado em Engenharia Civil) - Departamento de Engenharia Civil, Universidade Federal de Santa Catarina, Florianópolis, 2006. 